ojs.uv.es/index.php/qfilologia/index

Rebut: i6.06.2020. Acceptat: 07.08.2020

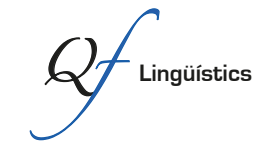

Per a citar aquest article: Alonso Rey, Rocío. 2020. "Metodología de la enseñanza de portugués a

hablantes de español: un marco y una propuesta de planificación del tratamiento de las competencias

lingüísticas". Quaderns de Filologia: Estudis Lingüístics XXV: 99-I20.

doi: $10.7203 /$ Q.25.I907I

\title{
Metodología de la enseñanza de portugués a hablantes de español: un marco y una propuesta de planificación del tratamiento de las competencias linguíísticas
}

\author{
Portuguese Language Teaching to Spanish Speakers: a framework and a planning \\ proposal on the treatment of linguistic competences
}

\author{
Rocío Alonso Rey \\ Universidad de Salamanca \\ rocioalonsorey@usal.es
}

Resumen: Las propuestas metodológicas en el campo del portugués para hablantes de español resultan demasiado generales o presentan moldes demasiado rígidos para su aplicación en el aula. En este trabajo se desarrolla un modelo de organización de las secuencias para el tratamiento de aspectos competenciales. El marco operacional presentado se basa en las principales especificidades metodológicas del campo y es compatible con diferentes perspectivas y tipos de enseñanza.

Palabras clave: portugués lengua extranjera; portugués para hablantes de español; metodología de la enseñanza; secuenciación de la clase.

Abstract: The methodologycal proposals in the field of Portuguese for Spanish speakers are too wide or too restricted to apply in the classroom. This paper presents a model to organize the sequences based on competencial contents. This operational frame is based on the main methodological aspects of field and allows different perspectives and teaching orientations.

Keywords: Portuguese as foreign language, Portuguese for Spanish speakers, teaching methodology, classroom sequences. 


\section{Introducción}

En el marco de la didáctica del Portugués como Lengua Extranjera, la especialidad del Portugués para Hablantes de Español (PHE) no ha encajado con facilidad dentro del paradigma comunicativo en la enseñanza de lenguas. Aunque, en general, se comparten y asumen los principios del enfoque comunicativo, hay consenso en que un enfoque comunicativo puro, centrado en el significado, resulta insuficiente para este tipo de aprendices (Carvalho, 200I; Ferreira Montero, I996; Grannier, 20I5; Stoivecek, 20I7). Diversos autores han defendido la necesidad de una metodología específica al observarse que los hablantes de español poseen un conjunto de características propias que distinguen su proceso de aprendizaje del de hablantes de otras lenguas tipológicamente más distantes (Almeida, I995; Alonso, 2012; Simões, Carvalho \&Wiedemann, 2004; Wiedemann \& Scaramucci, 2008). Los manuales de PHE de las últimas décadas han tratado de adaptarse a esas particularidades ofertando materiales centrados en contenidos gramaticales diferenciales (Arregui \& Silva, 20I2; Simões, 2008), manuales de orientación gramatical comunicativizados (Bateman, Mattos, Brasileiro \& Knapp, 20I7; Ferreira \& Zagalo, 2003) o manuales basados en tareas (Dias, 2009).

Además de estas propuestas prácticas, diversos especialistas han contribuido a la metodología de PHE con propuestas que podríamos denominar teóricas, de carácter general, derivadas de estudios de adquisición o basadas en aspectos generales del aprendizaje de lenguas (Almeida, I995; Bateman, 2017; Carvalho \& Child, 2018; Grannier, 2002, 2015, entre otros).

Sin embargo, estas aportaciones no siempre son fáciles de llevar a la práctica por profesores que preparan sus clases y unidades didácticas. Las propuestas teóricas en ocasiones son muy generales y no especifican lo suficiente los tipos de actividad, procedimientos o técnicas a utilizar. Por otra parte, las propuestas prácticas de los manuales y libros de texto tienen formatos cerrados, con moldes demasiado rígidos, y su orientación didáctica puede no adaptarse al currículo o la programación didáctica a seguir.

En este trabajo, tomando como referencia las orientaciones generales para una metodología de PHE, se realiza una propuesta de protocolo de planificación con un modelo de secuenciación específico para la especialidad. Este marco operativo permitiría al docente integrar las prácticas recomendadas en el aula de PHE y, al mismo tiempo, le ofrece la flexibilidad suficiente para que resulte compatible con diversos enfoques y modelos de enseñanza. 
Partiendo de una revisión razonada de una serie de orientaciones metodológicas fundamentales para la enseñanza PHE, se establecen una serie de pasos, a modo de protocolo de actuación, para la planificación y un modelo de secuenciación de aspectos competenciales ${ }^{\mathrm{I}}$. Se presentan, además, algunos tipos de actividades y procedimientos básicos para su desarrollo.

\section{Aspectos metodológicos del PHE}

Los aspectos que han acaparado gran parte de la discusión en torno a la metodología PHE se pueden agrupar en dos grandes grupos, uno relacionado con los contenidos y otro con los procedimientos. En lo que se refiere a los primeros, se considera que la enseñanza de PHE debe estar centrada en un conjunto de puntos críticos (Grannier, 2002; Bateman, 20I7) entendidos como los aspectos lingüísticos en los que divergen los sistemas de ambas lenguas. Estas divergencias son puestas de manifiesto mediante el análisis contrastivo (AC) de los dos sistemas. En relación con los procedimientos, se ha planteado la necesidad de que el aprendiz desarrolle o potencie habilidades estratégicas y de aprendizaje específicas tales como la conciencia de la diferencia o la conciencia metalingüística, consideradas cruciales para optimizar el proceso de aprendizaje.

\subsection{Contenidos}

El protagonista absoluto en las consideraciones sobre contenidos en el PHE es, sin duda, el análisis contrastivo (AC). El recurso al análisis contrastivo goza de una larga tradición en la especialidad (v. Alonso, 20I5; Carvalho, 2002; Rabassa, 2010). Rechazando la perspectiva conductista del aprendizaje de lenguas con que se asociaba inicialmente, el AC es aceptado como una herramienta útil para la provisión de estructuras lingüísticas divergentes (Azevedo, I978; Carvalho, 2002; Simões, Carvalho \& Wiedemann, 2004; Grannier, 2002; Lombello, El-Dash \& Baleeiro, I983; Wiedemann \& Scaramucci, 2008). Su papel sería, por tanto, el de ofrecer una especie de repertorio de puntos diferenciales que deben ser tratados en el aula.

\footnotetext{
${ }^{\mathrm{I}}$ Se entiende por aspectos o contenidos competenciales los referidos a las competencias comunicativas del MECR o las establecidas, en relación con estas, en los diferentes currículos o programaciones didácticas.
} 
Asimismo, se ha considerado que esta estrategia contrastiva de selección de contenidos gramaticales permite prescindir de la explicación de rasgos o reglas comunes en ambas lenguas (Bateman, 20I7; Pletch, I993; Santos I998; Simões \& Kelm, I99I). El tratamiento de los aspectos convergentes puede no aportar nada a la progresión de aprendizaje e, incluso, puede resultar desmotivador para el aprendiz. El planteamiento de los autores consiste en, a grandes rasgos, dedicar menos tiempo a los aspectos comunes y que necesitarían menos práctica, ajustando el desarrollo de la clase a las necesidades de los aprendices HE.

Sin embargo, algunos especialistas previenen contra algunos excesos o interpretaciones radicales de esta estrategia, rechazando la provisión de estructuras sin contextualizar (Santos \& Silva, 2004) o matizando que no se trata de prescindir totalmente de los puntos convergentes (Child, 20I3), ya que los aprendices necesitan tiempo para interiorizar la gramática y el vocabulario.

La perspectiva que aquí se defiende parte de que la clave para considerar la adecuación de la estrategia contrastiva en la enseñanza de PHE está en considerar qué se asume y qué implica. Inicialmente el uso del contraste se basa en el presupuesto de que los desvíos encontrados en el proceso de enseñanza/ aprendizaje de PHE obedecen mayoritariamente a los efectos de la influencia del español y que, por tanto, se puede asumir que tal influencia opera como mecanismo de aprendizaje y uso de la lengua. Mediante la instrucción específica de las formas divergentes se contrarrestarían sus efectos negativos, la fijación de formas incorrectas y el uso de formas desviadas.

Ahora bien, los errores de interferencia, fruto de las divergencias entre ambas lenguas, no son los únicos desvíos en la producción de los aprendices HE. Aunque tradicionalmente el PHE, tanto desde la perspectiva del aprendizaje como de la enseñanza, se haya centrado en este tipo de dificultades, la influencia de la $\mathrm{LI}^{2}$ por sí misma no es suficiente para dar cuenta del proceso de aprendizaje en su conjunto y, en consecuencia, la estrategia contrastiva supone una acción parcial y limitada a una parte de la construcción del conocimiento, aquella en la que operarían procesos de transferencia que conducen a formas desviadas. Por tanto, los efectos de la instrucción en la

\footnotetext{
${ }^{2}$ Se utiliza Li en referencia a la lengua de partida, el español. Se interpreta aquí, además, Li en sentido amplio, pudiendo tratarse tanto de la lengua materna del aprendiz como de otra segunda lengua que funciona como lengua de referencia o lengua interpuesta, a partir de la que operaría la transferencia. Sobre el estatuto de "aprendiz HE”, véase Johnson (2004) en Simões et alii.
} 
divergencia solo serán útiles si efectivamente los desvíos en el aprendizaje y uso de la lengua obedecen a la influencia de la Li o transferencia.

Aun reconociendo esta importante limitación, hay que tener en cuenta que la divergencia revela un punto donde tiene que haber una reestructuración del sistema, esto es, donde se tienen que desarrollar nuevas formas, valores o asociaciones, en definitiva, aspectos sujetos a aprendizaje. Aunque no se pueda anticipar el mecanismo que se utilizará, si será problemático o qué tipo de desvío se producirá, el sistema debe entrar en "modo aprendizaje" y proceder a la fijación e integración del nuevo conocimiento.

Desde esta perspectiva los resultados del análisis contrastivo sí pueden ser asumidos como objetivo de aprendizaje, no porque vaya a haber "interferencia" del español, sino por su estatuto de conocimientos necesarios para la construcción de la lengua objeto (LO). Dicho de otra manera, los establecidos por contraste son conocimientos no disponibles para el aprendiz en los que debe ser instruido.

Una segunda cuestión es qué se entiende por aspecto diferencial o punto crítico y qué implica. Al hablar de punto crítico, se asume que, en los diferentes niveles lingüísticos o componentes de la lengua, existen un conjunto de formas, un conjunto de funciones (entendidas estas como significados o valores gramaticales) entre los cuales se establecen relaciones o asociaciones. Los puntos críticos serían aspectos puntuales divergentes (sea en el valor, sea en la forma o en las asociaciones establecidas) en una red de relaciones coincidente. Esto implica que el sistema lingüístico tiene que tener configurada previamente dicha red, esto es, tiene que formar parte de su interlengua. En consecuencia no se puede asegurar la eficacia de la instrucción de un aspecto divergente si la base de convergencia no está establecida. En este sentido es necesario que el aprendiz tenga acceso a los aspectos similares entre las dos lenguas para construir o incorporar las divergencias. Se trata, en definitiva, de mostrar el funcionamiento y organización de la LO y, sobre esa base, incorporar las diferencias.

Planteada en estos términos, resulta, prácticamente, la estrategia contraria al AC clásico, que partía de la Li para llegar a la L2, eliminando hábitos "nocivos”. Aquí se parte de la L2 y se desmonta la dependencia de la Li. Metodológicamente, en la planificación, esto se traduce en la propuesta de introducir una fase contrastiva en la creación de secuencias de clase. 


\subsection{Procedimientos}

A la hora de llevar los contenidos al aula, varios autores inciden en la necesidad de que los aprendices HE usen procesos conscientes para la optimización del proceso de aprendizaje, concretamente procesos de dos tipos, relacionados con la conciencia de la diferencia y la conciencia metalingüística.

El tratamiento basado en la conciencia de la diferencia se basa en la observación de que el aprendiz no percibe o no retiene las diferencias entre ambos sistemas, lo que se hace visible en la producción, con numerosos desvíos relacionados con la interferencia del español. Así, se considera que el alumno debe ser consciente de esa diferencia (Almeida, I995; Grannier, 2002) como paso previo a separar lo que pertenece a una y otra lengua. Los procedimientos o técnicas para poner de relieve esas divergencias varían según los autores, desde presentaciones con contraste explícito de los sistemas (Alonso, 20I2; Child, 20I3) a actividades prácticas que incidan en los aspectos diferenciales (Grannier, 2002; 20I5), pasando por tratamientos del error en la producción (Almeida, 2004) o planteamientos más amplios como la propuesta heterodoxa de Grannier (2002).

La conciencia de la diferencia es interpretada aquí en términos de conocimiento interlingüístico, esto es, el aprendiz debe saber que determinadas formas, valores y asociaciones difieren en ambas lenguas. Esta afirmación es relevante ya que la transferencia positiva o facilitativa, el aprovechamiento de la Li (u otra lengua próxima) para la construcción de la LO, es un mecanismo de aprendizaje y construcción del nuevo sistema extremadamente potente en el caso de los HE y le impulsa a prescindir de las divergencias. Desde la perspectiva de la enseñanza, esto se traduce en que el profesor deberá proporcionar los recursos necesarios para ese contraste (la fase contrastiva que se mencionaba en 2.I.) y plantear actividades en que se explote ese conocimiento contrastivo estratégicamente, es decir, que ayude a optimizar el aprendizaje y uso de la lengua. En esas actividades se debe utilizar algún tipo de procedimiento en que la conciencia de la diferencia ayude a minimizar el impacto negativo del español. En otras palabras, actividades que recojan formas de usar ese conocimiento interlingüístico para mejorar la producción y sirvan de entrenamiento en la detección de formas desviadas.

En lo que respecta a la conciencia metalingüística, varios estudios han puesto de relieve que algunos HE no explotan el conocimiento metalingüístico en actividades de producción (Carvalho, Freire \& Silva, 20ıо; Carvalho \& Silva, 2008) o como estrategia de control de la producción y corrección de errores (Alonso, 20I6), guiándose más por un conocimiento implícito de la 
LO basado en una interlengua deficitaria que por un conocimiento explícito de su funcionamiento. Parece que estos aprendices presentan un estilo de aprendizaje y uso de la lengua que prescinde del conocimiento explícito del sistema y de la reflexión metalingüística.

No se han propuesto técnicas o procedimientos específicos, pero se entiende que el aprendiz deberá ser confrontado intencionalmente con el modo de funcionamiento de la LO (que deberá ser retenido como conocimiento explícito) y deberá realizar actividades prácticas que estimulen el uso de ese conocimiento.

En otro orden de cosas, la conciencia de la diferencia y la conciencia metalingüística son procesos conscientes, de atención a la forma, que generalmente se relacionan con la formulación explícita de reglas lingüísticas. Estas reglas y el funcionamiento del sistema en general se presentan al alumno mediante un procedimiento de exposición directa o presentación frontal. Es la técnica adoptada en la mayoría de los manuales o sugerida en algunas orientaciones metodológicas para la enseñanza de PHE (Bateman, 20I7). Una alternativa es desarrollar ese conocimiento mediante actividades de inferencia (Grannier, 20I5; Alonso, 20I5) en que el alumno descubre por sí mismo los contenidos y puede llegar a formular explícitamente esos conocimientos. Estas dos técnicas, exposición e inferencia son contempladas como procedimientos posibles en la propuesta.

\section{Propuesta metodológica}

\subsection{Modelo de secuencia para la planificación}

En Alonso (en prensa) se esboza un modelo para la secuenciación de los contenidos de la clase de PHE y lenguas próximas que sirve de base para la integración de la traducción pedagógica en la enseñanza de PHE. Ese modelo centrado en el tratamiento de contenidos competenciales se desarrolla y justifica aquí como propuesta general para la integración de otros tipos de actividad y procedimientos determinados por las orientaciones metodológicas del campo presentadas en el apartado anterior.

A grandes rasgos, se trata de un modelo de síntesis, basado en propuestas de secuenciación como el modelo clásico o modelo PPP3 (Sánchez, 20I4),

\footnotetext{
${ }^{3}$ Siglas que corresponden a las fases que contempla: presentación, práctica y producción.
} 
planteamientos comunicativos ${ }^{4}$ (Cerrolaza \& Cerrolaza, I999) o de la enseñanza mediante tareas5 (Nunan, I998, 2006; Skehan, 2003 o Zanón, I999). Se organiza en 3 grandes fases o rutinas (Tabla I) que se aplican a cada contenido competencial trabajado en la sesión e incluye una subrutina específica en que se introduce el contraste entre las lenguas.

\begin{tabular}{|l|l|l|}
\hline \multicolumn{1}{|c|}{ RUTINA } & \multicolumn{1}{|c|}{ SUBRUTINA } & \multicolumn{1}{c|}{ ÁREA DE ACTUACIÓN } \\
\hline \multicolumn{4}{|l}{ 1. Localización } & Situación comunicativa \\
\hline \multirow{4}{*}{ 2. Desarrollo } & 2.1. Observación & Contexto lingüístico \\
\cline { 2 - 3 } & 2.2. Explicitación & Conocimiento de la LO \\
\cline { 2 - 3 } & 2.3. Contraste & Conocimiento interlingüístico \\
\hline \multirow{3}{*}{ 3. Práctica } & 3.1. Uso & $\begin{array}{l}\text { Uso del conocimiento en contextos lingüísticos } \\
\text { significativos }\end{array}$ \\
\cline { 2 - 4 } & 3.2. Corrección & Provisión de feedback \\
\hline
\end{tabular}

Tabla I. Modelo de secuenciación de contenidos competenciales

En la primera fase, Localización (I), se muestra una situación comunicativa en la que ocurre el aspecto lingüístico a trabajar. Se trata de mostrar un entorno comunicativo o contexto de uso en que se verifica ese elemento ${ }^{6}$.

La rutina de Desarrollo (2) corresponde al tratamiento que se da al conocimiento que el alumno debe incorporar: es la fase en la que el alumno desarrolla nuevos conocimientos lingüísticos. Este tratamiento se compone a su vez de tres subrutinas: en Observación (2.I.) se presentan al alumno casos de uso de las unidades, esto es, las unidades se muestran en un contexto lingüístico con valor comunicativo/significativo a partir de la situación previamente planteada. La subrutina de Explicitación (2.2.) consiste en la formulación del conocimiento lingüístico, esto es, se trata de la fase en que se analiza el funcionamiento de la LO (reglas gramaticales, unidades léxicas y sus significados, etc.). La subrutina de Contraste (2.4.) corresponde a la comparación entre la LO y la LI. Justificada por criterios metodológicos en el caso de PHE y lenguas próximas, sirve para desarrollar un conocimiento interlingüístico de contraste entre las lenguas. Finalmente, la fase de práctica (3) es el momento de la clase en que el aprendiz usa el conocimiento anterior en operaciones

\footnotetext{
${ }^{4}$ Fases de preparación, presentación, conceptualización, práctica y producción.

${ }^{5}$ Fases de pretarea, tarea y postarea.

${ }^{6}$ La justificación y más detalles sobre esta fase se pueden encontrar en los modelos de planificación de referencia.
} 
lingüísticas como parte de contextos lingüísticos significativos (3.I.) y recibe el feedback del profesor en la corrección (3.2.)

\subsection{La construcción de una secuencia a partir del modelo introduciendo los aspectos metodológicos de contenido y procedimiento del PHE}

Se asume que la planificación de una sesión se puede desarrollar en los siguientes pasos: selección de contenidos ${ }^{7}$, secuenciación, selección de tipos de actividades y elaboración de materiales.

\begin{tabular}{|l|l|l|}
\hline \multirow{2}{*}{ CONTENIDOS } & Paso 1 & Selección de contenidos \\
\cline { 2 - 3 } & Paso 2 & Secuenciación de contenidos \\
\hline \multirow{2}{*}{ ACTIVIDADES } & Paso 3 & Determinar tipos de actividades y sus procedimientos \\
\cline { 2 - 3 } & Paso 4 & Elaborar los materiales (creación o recopilación) \\
\hline
\end{tabular}

Tabla 2. Pasos de la planificación de una secuencia de clase

El punto de partida de la planificación de la sesión consiste en determinar los contenidos específicos de la unidad que se quieren trabajar en el aula. Se consideraría qué aspectos y estructuras específicas se presentarán al alumnado así como los objetivos y tema de las actividades comunicativas a realizar ${ }^{8}$.

El segundo paso consistiría en organizar esos bloques de contenido típicamente en una plantilla (tabla 3), determinando el orden de tratamiento en la secuencia de clase completando la columna de correspondiente (columna I, Contenido, en la tabla 3).

A continuación se aplicaría el modelo de secuencia a cada contenido competencial9 (columna 2, fase, en la tabla 3) y, a continuación, se determinaría el tipo de actividad a realizar (columna 3, actividad, en la tabla 3).

\footnotetext{
7 Se utiliza aquí "contenido" en sentido amplio e incluiría tanto aspectos competenciales como las actividades comunicativas del MECR (comprensión, producción, interacción, mediación). Incluiría también los objetivos específicos que ofrecen algunos documentos curriculares y programaciones.

${ }^{8}$ Este protocolo general se utiliza en la disciplina de Didáctica de la Lengua Portuguesa, del Grado en Estudios Portugueses y Brasileños (USAL) y este paso se plasma un documento con la especificación de contenidos/objetivos, acompañado de la plantilla de planificación que se comenta a continuación.

9 El modelo de secuencia para las actividades comunicativas queda fuera del alcance de este trabajo y se completa con una secuencia básica tomada de la enseñanza por tareas.
} 


\begin{tabular}{|l|l|l|l|}
\hline \multicolumn{1}{|c|}{ CONTENIDO } & \multicolumn{2}{|c|}{ FASE } & ACTIVIDAD \\
\hline \multirow{4}{*}{ Competencia "X" } & \multicolumn{2}{|c|}{ 1.1. Localización } & \\
\cline { 2 - 4 } & & 1.2.1. Observación & \\
\cline { 2 - 4 } & & 1.2.2. Explicitación & \\
\cline { 2 - 4 } & 1.2.3. Contraste & \\
\cline { 2 - 4 } & 1.3. Práctica & \\
\hline \multirow{3}{*}{$\begin{array}{l}\text { Actividad comuni- } \\
\text { cativa "Y" }\end{array}$} & 2.1. Pretarea & \\
\cline { 2 - 4 } & 2.2. Tarea & \\
\cline { 2 - 4 } & 2.3. Postarea & \\
\hline
\end{tabular}

Tabla 3. Modelo simplificado de plantilla de planificación de la clase con contenido competencial "X" y destrezas " $\mathrm{Y}$ "

Una vez completada plantilla de planificación, se elaboran las actividades ya sea creando materiales propios, ya sea seleccionándolas de materiales preexistentes (libros de texto, recopilaciones de ejercicios, etc.). Sobre este modelo de planificación se introducen y comentan a continuación las especificidades metodológicas del PHE en relación con los contenidos competenciales.

\subsubsection{Contenidos}

La selección y establecimiento de los contenidos y objetivos es un aspecto clave en la planificación de la clase para hablantes de español, ya que en este punto el docente puede utilizar el análisis contrastivo como herramienta para determinar posibles áreas de dificultad. De manera que, al tiempo que se establecen los aspectos de la LO que se van a mostrar al aprendiz, se compara con el funcionamiento en la lengua de referencia (español en este caso). Se trata de establecer el funcionamiento de un determinado aspecto lingüístico de la LO y contrastarlo con la LI, de manera que se recojan tanto las áreas de divergencia como las de convergencia. Los aspectos divergentes suponen conocimientos nuevos, no disponibles, que deben ser introducidos en el nuevo sistema; los aspectos convergentes son conocimientos disponibles desde la LI pero necesarios para poder encuadrar las diferencias.

Otro tipo de información relevante en este punto es la que aportan los análisis de errores (AE). La consulta de este tipo de trabajos y la observación de los 
errores en el aula proporcionan pistas valiosas sobre el proceso de aprendizaje y uso así como de las dificultades que los alumnos encuentran en ese proceso. Esta indagación y reflexión previa permite al profesor determinar o modificar los procedimientos y técnicas específicos en el diseño de actividades.

En conclusión, este modelo de planificación introduce además de los planteamientos habituales en la metodología de enseñanza (tabla 4), planteamientos específicos para la metodología PHE que toman en consideración las aportaciones del análisis contrastivo y el análisis de errores.

\begin{tabular}{|l|l|l|}
\hline \multicolumn{1}{|c|}{ SELECCIÓN } & \multicolumn{1}{|c|}{ Metodología general } & \multicolumn{1}{c|}{+ + Metodología PHE } \\
\hline \multirow{2}{*}{$\begin{array}{l}\text { Contenido } \\
\text { competencial } \\
\text { "X" }\end{array}$} & $\begin{array}{l}\text { Funcionamiento de "X" en } \\
\text { portugués }\end{array}$ & $\begin{array}{l}\text { Funcionamiento de "X" en } \\
\text { español }\end{array}$ \\
\cline { 2 - 3 } & $\begin{array}{l}\text { Aspectos divergentes y } \\
\text { convergentes (AC) } \\
\text { Desvíos más frecuentes (AE) }\end{array}$ \\
\hline
\end{tabular}

Tabla 4. Planteamientos en la metodología estándar y planteamientos a sumar en la metodología PHE para la selección de contenidos

\subsubsection{Las actividades}

Una vez establecidos los contenidos y analizados los aspectos diferenciales y problemáticos para los aprendices, se aplica sobre la plantilla el modelo de secuencia para cada componente y se comenzaría con la selección de tipos de actividades y procedimientos para cada uno de los contenidos.

\begin{tabular}{|l|l|l|}
\hline \multicolumn{1}{|c|}{ SECUENCIA } & \multicolumn{1}{|c|}{ Metodología general } & \multicolumn{1}{c|}{+ + Metodología PHE } \\
\hline $\begin{array}{l}\text { Contenido } \\
\text { competencial } \\
\text { "X" }\end{array}$ & $\begin{array}{l}\text { Aplicación del modelo de } \\
\text { secuencia }\end{array}$ & $\begin{array}{l}\text { Modelo de secuencia con } \\
\text { subrutina contrastiva }\end{array}$ \\
\hline
\end{tabular}

Tabla 5. Planteamientos en la metodología estándar y planteamientos que hay que sumar en la metodología PHE para la secuenciación de contenidos.

De las tres subrutinas indicadas, la fase de localización es común en otros modelos habitualmente usados en la preparación de secuencias y no requiere de especificaciones ad hoc en el marco PHE. 


\subsubsection{La fase de desarrollo en la metodología PHE. Las subrutinas de observación y explicitación}

La fase de observación consiste en la presentación de casos de uso contextualizado del elemento que trabajar (ejemplos seleccionados o un texto ${ }^{\mathrm{I}}$ ) y se justifica porque un proceso inductivo de aproximación al conocimiento resulta más adecuado para la explicación de estos aspectos, es decir, las reglas o explicaciones se entienden mejor si se ven primero los casos de uso (la formas utilizadas en un contexto lingüístico significativo), en lugar de operar en abstracto y presentar los casos o ejemplos al final. Esto, que puede resultar muy obvio para aspectos como el léxico, no siempre se ha asumido con los aspectos gramaticales. Además, la presentación de casos es necesaria si se utiliza un procedimiento de inferencia en la fase de explicitación.

Por otra parte, la fase de explicitación permite desarrollar el conocimiento explícito (de ahí su nombre) del funcionamiento del sistema. Se trata de visibilizar un determinado aspecto del sistema de la LO y contribuir a su integración en la competencia del aprendiz. Esto quiere decir que se deben aportar, para las diferentes unidades lingüísticas, informaciones relativas a tres grandes clases de material lingüístico: (a) formas o estructuras, (b) significados o valores y (c) determinadas asociaciones de las anteriores (en el sentido de reglas o formas de organización) que determinan la arquitectura de la lengua.

Si se toman como ejemplo, dentro de la competencia gramatical, el presente de indicativo y, como contenidos específicos, los verbos regulares y los verbos con alternancia vocálica de la $3{ }^{\text {a }}$ conjugación, se trataría de:

(a) valor de Presente

(b) formas regulares (paradigma flexivo)

(c) formas con alternancia (regla de alternancia asociada a $<$ e,o $>$ en la tercera conjugación tipo ferir, dormir).

Téngase en cuenta que en esta propuesta se considera necesario que haya una presentación explícita de los contenidos que permita la construcción de un conocimiento metalingüístico de la LO y que se incluyan los elementos convergentes en ambas lenguas, aunque sea de manera sucinta.

\footnotetext{
to Si en un bloque anterior se ha trabajado, por ejemplo, la comprensión con un texto. La fase de localización puede ser suprimida y la subrutina de observación se puede centrar en los casos presentes en ese texto.
} 
Los procedimientos para aproximar esos conocimientos a los aprendices son fundamentalmente de dos tipos, la inferencia y la exposición. La inferencia consiste en que el alumno descubra por sí mismo las asociaciones, formas y valores a partir de instrucciones orientadoras, que van desde preguntas del profesor hasta actividades más elaboradas con la ayuda de materiales específicos. La alternativa clásica es la exposición, con la explicación del profesor de los aspectos correspondientes o provista por los materiales.

En estos dos procedimientos se asume que primero es necesario desarrollar el conocimiento (fase 2) y, a partir de él, se realizan operaciones lingüísticas, se practica el elemento (fase 3). Una tercera posibilidad es invertir ese orden: solicitar una determinada operación lingüística (o tarea) que implica un nuevo elemento/contenido (fase 3 ) y retroalimentar con los conocimientos necesarios (fase 2) para ajustar el producto final ${ }^{\text {II }}$. Este tipo de procedimiento, que llamaremos aquí de exposición diferida ${ }^{\mathrm{i2}}$, tiene la ventaja de que sensibiliza al aprendiz sobre su dominio de los recursos específicos y crea una necesidad que se explota en la explicación subsiguiente.

La decisión de utilizar un procedimiento específico depende de varios factores y puede responder a diferentes criterios. Desde el punto de vista de la metodología PHE es relevante considerar la comparación entre los dos sistemas. Aunque en este punto no se trata de establecer explícitamente ese contraste, el resultado de la comparación puede ayudarnos a decantarnos por un tipo u otro de procedimiento. Así, por ejemplo, para los elementos divergentes formales resulta indicado el procedimiento de inferencia, ya que obliga al aprendiz a dirigir su atención a estos aspectos que normalmente tiende a ignorar (al menos en etapas tempranas) en favor de los significados.

En el lado opuesto del espectro se sitúan algunos aspectos semánticos o funcionales divergentes, que no son visibles en el contexto, por ejemplo, formas como população en el dominio léxico ${ }^{13}$. También determinadas asociaciones de forma/función no coincidentes en la lengua de referencia cuyas marcas o valores no son trazables pueden resultar más fáciles de comprender en una presentación frontal. Por ejemplo, en el caso de los verbos con alter-

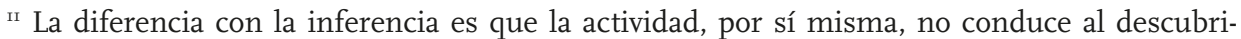
miento de los aspectos lingüísticos necesarios o no está diseñada para con esa intención. Las actividades de inferencia se diseñan con preguntas o pistas que guían el descubrimiento. En este procedimiento, la explicación es presentada a posteriori por el profesor.

${ }^{12}$ Este procedimiento es también denominado "garden path technique" (Nunan, 2003).

ז3 "Población”, en español, se refiere a un conjunto de personas o a un lugar, el equivalente en portugués solo al conjunto de personas.
} 
nancia vocálica, la presencia de <e, o> en la penúltima sílaba no es una marca en la que vaya a reparar con facilidad el alumno y una actividad que guíe hacia ese aspecto puede acabar resultando más complicada de seguir que la explicación frontal.

Por otra parte, un aspecto convergente puede ser resuelto, de ambas formas mediante una formulación sencilla como exposición (o presente de Indicativo é o tempo verbal do agora....) o como inferencia, mediante una simple pregunta (que tempo verbal é este e para que serve?)

Una última opción que cabe considerar es la mezcla de procedimientos de exposición e inferencia. En el caso del presente de indicativo, se podría optar por:

\begin{tabular}{|l|l|l|}
\hline \multicolumn{1}{|c|}{ CONTENIDO } & \multicolumn{1}{|c|}{ PROCEDIMIENTO } & \multicolumn{1}{c|}{ TIPO DE ACTIVIDAD } \\
\hline (a) valor de presente & Inferencia & Pregunta del docente \\
\hline (b) formas regulares & Inferencia & $\begin{array}{l}\text { Localizar las formas y completar } \\
\text { un cuadro }\end{array}$ \\
\hline (c) formas con alternancia & Exposición & Presentación frontal \\
\hline
\end{tabular}

Tabla 6. Ejemplo de selección de procedimientos y tipos de actividad para Presente de Indicativo (formas regulares y con alternancia vocálica gráfica)

\subsubsection{Subrutina de contraste}

La fase de contraste es un elemento ad hoc del modelo y de la metodología PHE y su objetivo es mostrar al aprendiz el foco de divergencia entre las lenguas, ayudándole a separar los sistemas. No debe ser entendida como una reflexión lingüística o filológica extensa, sino más bien como un apunte rápido y preciso, una llamada de atención sobre aspectos diferenciales entre las dos lenguas. El fin último no es que el aprendiz sea capaz de hacer comparaciones interlingüísticas (aunque en un contexto de enseñanza universitaria pueda contemplarse también como un objetivo o una competencia a desarrollar), sino hacer visibles las divergencias entre los sistemas. La confrontación con estos elementos diferenciales que, en ocasiones, el aprendiz puede pasar por alto o le pueden resultar más difíciles de dominar, funciona como una especie de bandera roja, que alerta al aprendiz.

En este punto de la planificación se recuperan las informaciones recabadas en el paso I para determinar el procedimiento y diseñar la actividad correspondiente. Los procedimientos aplicables serían los indicados en el apartado de explicitación: exposición, con una presentación directa del profesor de viva 
voz o con el apoyo de materiales, o bien, por un proceso de inferencia, en el que el alumno realiza la operación de contraste y reflexiona sobre el funcionamiento de la lengua de referencia ${ }^{\mathrm{T}}$.

Por otro lado, aunque la subrutina de contraste aparece situada en última posición, se concibe como una rutina flexible, prescindible incluso, que se puede integrar en las subrutinas anteriores. De hecho el contraste puede realizarse antes de la explicitación del elemento correspondiente de la LO o en paralelo. Si tenemos en cuenta que el aprendiz realiza inconscientemente este tipo de comparaciones cuando entra en contacto con la LO, a partir de los casos de la fase de observación se puede acompañar ese proceso y desarrollar el contraste en ese momento.

Además, determinados contenidos como, por ejemplo, un conjunto de formas léxicas, que se introducen en la fase de observación pueden ser presentados y contrastados en LO unidad a unidad, por ejemplo, en el léxico de las comidas: se trataría de hacer un rápido apunte sobre la forma almoçar en contraste con almorzar.

En términos generales, el contraste es suprimible cuando es claramente visible (formas totalmente divergentes como la palabra bolacha, acompañada de un paquete de galletas, no necesita mayores explicaciones) pero no en el caso de formas parcialmente divergentes (almoçar-almorzar) o cuando implica aspectos funcionales o de organización del significado. En último término para que el contraste sea efectivo tiene que contribuir al proceso de aprendizaje, esa aportación es efectiva si consigue que el aprendiz perciba y sea consciente de los aspectos diferenciales.

Cabe señalar por último el valor añadido de la subrutina contrastiva en términos de competencia de aprendizaje: el aprendiz se habitúa a realizar unas operaciones estratégicas que le ayudarán en su progresión de aprendizaje autónomo a lo largo de su vida (reparar en pequeñas diferencias formales o no asumir correspondencias I a I) y adquiere los conocimientos necesarios para desarrollar instrumentos de control de la producción (que veremos en el siguiente apartado), sin olvidar que este tipo de conocimientos son necesarios para la mediación lingüística y cultural.

\footnotetext{
${ }^{14}$ En este punto es probable que los diferentes tipos de HE presenten diferencias: los hablantes nativos suelen ser menos conscientes del funcionamiento de la lengua que los HE con otras lenguas maternas que han recibido instrucción formal y han sido confrontados explícitamente con ese funcionamiento en su aprendizaje.
} 


\subsubsection{Fase de prácticas}

El objetivo en la fase de prácticas se puede interpretar como convertir un conocimiento declarativo en conocimiento instrumental, avanzar en el camino que va de saber algo a ser capaz de usarlo. La fase de práctica, en este modelo, crea entornos comunicativos para realización de operaciones con el objeto explicitado $^{15}$. Su finalidad no es desarrollar una actividad comunicativa (en el sentido del MECR), sino que se centra en el dominio o interiorización del aspecto seleccionado. Si se toma como ejemplo el léxico y se han presentado formas y significados, las operaciones posibles serán recuperar la forma que corresponde a un determinado significado o bien reconocer el significado que corresponde a esa forma.

Si se toma como ejemplo la gramática y se han trabajado las formas de determinados verbos irregulares en un tiempo determinado, las operaciones serán recuperar (y reconocer) las formas explicitadas.

En algunos manuales y planificaciones de profesores en formación se pueden observar secuencias en las que se pasa de la explicitación de un contenido a una actividad comunicativa de producción, sin fase práctica (en particular para el léxico). Se considera que con ese contacto es suficiente, postergando la fase práctica a la actividad comunicativa o tarea programada.

Esta es una opción perfectamente válida pero, en el caso del PHE, dados los problemas observados (la falta de dominio de las reglas y formas y tendencia a prescindir del conocimiento metalingüístico), la fase de prácticas es un punto crucial del proceso que puede servir para desarrollar actividades estratégicas que se sirvan del conocimiento metalingüístico e interlingüístico, al tiempo que se realizan las operaciones lingüísticas trabajadas.

\begin{tabular}{|l|l|l|}
\hline SECUENCIA & \multicolumn{1}{|c|}{ Metodología general } & \multicolumn{1}{c|}{+ Metodología PHE } \\
\hline $\begin{array}{l}\text { Fase } \\
\text { Práctica }\end{array}$ & $\begin{array}{l}\text { Actividades con operaciones } \\
\text { lingüísticas en contextos } \\
\text { significativos. }\end{array}$ & $\begin{array}{l}\text { Actividades que incentiven el uso del co- } \\
\text { nocimiento metalingüístico y del conoci- } \\
\text { miento interlingüístico (o consciencia de la } \\
\text { diferencia) }\end{array}$ \\
\hline
\end{tabular}

Tabla 7. Planteamientos en la metodología estándar y planteamientos a sumar en la metodología PHE en la fase de práctica

\footnotetext{
Is Otra de las propuestas metodológicas para el PHE que no se ha comentado aquí en detalle por razones de espacio es el uso de la instrucción con foco en la forma (v. Grannier, 20I5).
} 
En las actividades estratégicas con el conocimiento metalingüístico se trata de incentivar el uso de este conocimiento (reglas o formas de organización de la lengua) como forma de asegurar el output correcto. Se conciben como una forma de autofeedback correctivo o monitorización basada en los contenidos de la fase de explicitación al tiempo que realizan las operaciones lingüísticas. La introducción de este tipo de técnica es muy sencilla: en una actividad práctica normal (operacional) se introduce una instrucción estratégica. Por ejemplo, para los contenidos de formación del plural, en una actividad en que los alumnos tienen que determinar el número de productos que van a comprar (superior a I), escribiendo la forma que adoptarían esas palabras en plural, bastaría con añadir una instrucción del tipo: indica qué regla has aplicado.

El conocimiento interlingüístico o conciencia de la diferencia es también una herramienta valiosa y permite el desarrollo de estrategias de control como la anterior que funcionan bien en la fase de revisión en las actividades comunicativas de producción. Estas estrategias también pueden ser incentivadas en la fase de práctica de contenidos competenciales. La técnica de corrección de errores es un buen ejemplo de este tipo de actividades, en los manuales suele aparecer en el tratamiento de aspectos léxicos (v. Dias, 2009), pero se puede utilizar para otros contenidos competenciales. En Alonso (en prensa) se proponen algunas actividades utilizando la traducción pedagógica como recurso.

Se trata, en definitiva, de actividades que incentivan la fijación del conocimiento lingüístico e interlingüístico, desarrollan la capacidad de recuperarlos y usarlos para controlar la producción y contribuyen, en último término, a la autonomía del aprendiz.

\section{Conclusiones}

La propuesta de planificación y secuenciación de contenidos competenciales aquí presentada integra las principales orientaciones metodológicas que se han venido discutiendo en el campo del PHE. Su papel es meramente orientador y trata de poner a disposición del profesorado un modelo suficientemente flexible como para satisfacer las diferentes necesidades y realidades lingüísticas de la heterogénea categoría de hablantes de español así como de los diferentes currículos y programas que rigen la enseñanza.

Los puntos principales pueden ser considerados como adendas de la especialidad a la planificación estándar para el portugués u otra lengua extranjera 
(v. Modelo de planificación extendido en el Anexo para una visión de conjunto) y consisten en:

- la integración del AC como herramienta descriptiva en la selección de contenidos y su explotación en (a) la toma de decisiones sobre los procedimientos y (b) la fase de contraste;

- un modelo de secuenciación que incluye una fase de contraste,

- actividades en la fase de práctica que incentiven el uso del conocimiento metalingüístico e interlingüístico.

El AC es una herramienta válida en la metodología PHE siempre que se tenga en cuenta su papel y alcance en el proceso de enseñanza/aprendizaje. Los elementos diferenciales que se extraen del análisis deben ser considerados como objetos de aprendizaje, conocimientos necesarios para la construcción de la interlengua, desde un punto de vista estrictamente lingüístico. Sus posibilidades en relación con los mecanismos de transferencia de aprendizaje son limitadas y su efectividad no está verificada. Por otra parte, los aspectos diferenciales no se sustentan si el aprendiz no tiene acceso al conjunto de los datos: no se puede asegurar la eficacia del tratamiento de un aspecto divergente si la base de convergencia no está asegurada como parte de la LO.

La explicitación de aspectos interlingüísticos y el conocimiento del funcionamiento de la LO dotan al aprendiz de conocimientos que puede usar estratégicamente para controlar su producción y que pueden resultar especialmente útiles e incluso necesarios para la progresión del aprendizaje, además de ser necesarios para poder desarrollar capacidades de mediación lingüística y cultural. Asimismo, las actividades con uso de este tipo de conocimientos contribuyen al desarrollo de un aprendizaje autónomo.

En relación con el modelo de secuencia, es necesario hacer hincapié en que las rutinas y subrutinas más que puntos obligatorios e inamovibles deben ser tratados como aspectos que hay que considerar. Tal y como aquí se presentan son lo que, en lingüística generativa, se denomina una proyección máxima, que se puede verificar o no en la actuación o cuya posición puede variar. En la tabla $\mathrm{X}$ del anexo se presenta una lista de verificación que puede ser útil para los docentes que siguiendo otros modelos de planificación quieran comprobar si su planificación es consistente con las propuestas metodológicas PHE.

Finalmente cabe señalar que una de las limitaciones del modelo es que, en este punto de su desarrollo, no da orientaciones específicas para las actividades de comunicación o destrezas. Su ampliación en este sentido permitirá un 
diseño más completo de la clase desde la perspectiva del PHE. Por otro lado, los procedimientos indicados son sugerencias de uso y no recogen exhaustivamente todas las posibilidades. Su aplicación a competencias y aspectos seleccionados permitiría en el futuro una visión más amplia y precisa de los recursos metodológicos para el PHE.

\section{Bibliografía}

Almeida Filho, José Carlos P. I995. Uma metodologia específica para o ensino de línguas próximas? En Almeida Filho, José Carlos P. (org.) Português para estrangeiros interface com o espanhol. Campinas: Pontes, I3-2I.

Alonso Rey, Rocío. 20I2. La transferencia en el aprendizaje de portugués por hispanohablantes. Salamanca: Luso-Española de Ediciones.

Alonso Rey, Rocío. 20I5. Portugués para Hispanohablantes: aspectos teóricos y prácticos. Estudios Portugueses y Brasileños I2: 9-26.

Alonso Rey, Rocío. 20I6. Reconhecimento do erro na pronúncia na formação de professores não nativos falantes de espanhol: estudo de caso numa tarefa de autodiagnose. En Barros, Adriana L. Escobar Chaves \& Gomes, Nataniel dos Santos (org.) Anais do XI Congresso Brasileiro de Linguística Aplicada. Campo Grande: CBLA, I590-I605

Alonso Rey, Rocío. En prensa. El lugar de la traducción en la metodología de enseñanza del portugués a hablantes de español en niveles iniciales. Limite. Revista de Estudios Portugueses y de la Lusofonía I4.

Arregui, M. ${ }^{a}$ José \& Silva, José L. Lucimar. 20I2. Português para espanhóis I. Madrid: Agoralíngua.

Azevedo, Milton. 1978. Identifying Spanish Interference in the speech of learners of Portuguese. Modern Languages Journal 62(I-2): 18-23

Bateman, Blair. 20I7. Teaching Portuguese to Spanish Speakers. En Milleret, M. \& Risner, M. (ed.) Handbook for Portuguese Instructors. Roosevelt: Boa Vista Press, I95-213.

Bateman, B.; Mattos, M.; Brasileiro, M. \& Knapp, N. 20I7. Perspectivas: português para falantes de espanhol. Ronkonkoma: Linus Learning.

Carvalho, Ana M. 2002. Português para falantes de espanhol: perspetivas de um campo de pesquisa. Hispania 85(3): 597-608.

Carvalho, Ana M. \& Child, Michael 20I8. Expanding the multilingual repertoire: Teaching cognate languages to heritage Spanish speakers. En Potowski, Kim (ed.) Handbook of Spanish as a Minority/Heritage Language. Nueva York: Routledge, 42I-432.

Carvalho, Ana M.; Freire, Juliana L. \& Silva, Antônio J. B. da. 20ıo. Teaching Portuguese to Spanish Speakers: A Case for Trilingualism. Hispania 93(I): 70-75.

Carvalho, Ana M. \& Silva, Antônio J. B. da. 2008. O papel do conhecimento metalinguístico nos padrões de transferência no desenvolvimento da interlíngua 
e suas implicações pedagógicas. Portuguese Language Journal 3. http://www. ensinoportugues.org/wp-content/uploads/20II/05/Carvalho-da-Silvar.pdf

Cerrolaza, O. \& Cerrolaza, M. I999. Cómo trabajar con libros de texto. Madrid: Edelsa. Child, Michael. 2013. Language learning perceptions: The role of Spanish in L3 Portuguese acquisition. Portuguese Language Journal 7. http://www.ensinoportugues. org/wp-content/uploads/20I3/II/Child-20I3-Language-Learning-PerceptionsThe-Role-of-Spanish-in-L3-Portuguese-Acquisition.pdf

Dias, Ana C. 2008. Entre nós I. Lisboa: Lidel.

Ferreira, Itacira. I997. Interface português/espanhol. En Almeida Filho, J. C. P. de (ed.) Parâmetros atuais para o ensino de português língua estrangeira. Campinas: Pontes, I4I-I5I.

Ferreira Montero, Hélder J. \& Pereira Zagalo, F. I999. Português para todos I. Salamanca: Luso-Española.

Grannier, Danielle M., 2002. Uma proposta heterodoxa para o ensino de português a falantes de espanhol. En Júdice, Norimar (org.) Português para estrangeiros: perspectivas de quem ensina. Niterói: Intertexto, 57-80.

Grannier, Danielle M. 20I5. Revisitando a proposta heterodoxa. Estudios portugueses y brasileños I2: I6I-I76.

Lombello, L. C.; El-Dash, L. G. \& Baleeiro, M. A. I983. Subsídios para a elaboração de material didático para falantes de espanhol. Trabalhos em Lingüística Aplicada I, II7-I32.

Nunan, David. I998. El diseño de tareas para la clase comunicativa. Cambridge: CUP.

Nunan, David. 2003. Practical English Language Teaching. Singapur: McGraw-Hill.

Pletsch, K. García. I993. Portuguese for Spanish speakers: the usefulness of contrastive analysis. Romance Languages Annual 5: 495-50I.

Rabassa, Yamilka. 20ıo. A Emergência da Especialidade de Ensino de Português para Hispanofalantes no Brasil. Revista Helb 4(I). http://www.helb.org.br/index. php/revista-helb/ano-4-no-4-I2010/I43-a-emergencia-da-especialidade-deensino-de-portugues-para-hispanofalantes-no-brasil

Sánchez Pérez, Aquilino. 20I4. La enseñanza de idiomas en los últimos cien años. Madrid: SGEL.

Santos, P. I998. O ensino de português como segunda língua para falantes de espanhol: teoria e prática. Em Cunha, M. J. \& Santos, P. (org.) Ensino e pesquisa em português para estrangeiros. Brasília: Ednub, 49-57

Santos, Denise \& Silva, Glaúcia. 2004. Ensinando português para hispanofalantes: contrastes, transferências e a voz do aprendiz. En Simões, Antônio R. M.; Carvalho, A., \& Wiedemann, L. (org). Português para falantes de espanhol. Campinas: Pontes, I25-I5I.

Wiedemann, Lyris \& Scaramucci, Matilde V. R. 2008. Português para falantes de espanhol: ensino e aquisição. Campinas: Pontes.

Simões, Antônio R. M. 2008. Pois Não: Brazilian Portuguese Course for Spanish Speakers, with Basic Reference Grammar. Austin: University of Texas Press.

Simões, Antônio R. M. \& Kelm, Orlando. I99I. O processo de aquisição das vogais semi-abertas é, ó do português (brasileiro) como língua estrangeira. Hispania $74(3)$ : 654-665. 
Simões, Antônio R. M.; Carvalho, A. \& Wiedemann, L. 2004. Português para falantes de espanhol. Campinas: Pontes.

Skehan, P. 2003. Task-based instruction. Language Teaching 36(I): I-I4

Stovicek, T. 20I7. Approaches to Meeting the Needs of Spanish-Speaking Learners of Portuguese. Dialog on Language Instruction 27(I): 29-40.

Zanón, Javier (coord.). I999. La enseñanza del español mediante tareas. Madrid: Edinumen.

\section{ANEXO}

\begin{tabular}{|c|c|c|c|c|c|}
\hline & & \multicolumn{2}{|c|}{ METODOLOGÍA GENERAL } & \multicolumn{2}{|r|}{ METODOLOGÍA PHE } \\
\hline \multicolumn{6}{|c|}{ 1. Selección de contenidos/objetivos } \\
\hline \multicolumn{2}{|l|}{ Destrezas } & \multicolumn{4}{|c|}{ Competencias } \\
\hline \multicolumn{2}{|c|}{$\begin{array}{l}\text { ¿Qué actividades co- } \\
\text { municativas va a reali- } \\
\text { zar el alumno? }\end{array}$} & \multicolumn{2}{|c|}{$\begin{array}{l}\text { ¿Qué debe conocer y saber usar } \\
\text { el alumno? }\end{array}$} & \multicolumn{2}{|c|}{$\begin{array}{l}\text { ¿Cómo funciona ese aspecto en español? } \\
\text { ¿Cuáles son los aspectos diferentes y qué es } \\
\text { similar? } \\
\text { ¿Qué dificultades o errores son más frecuen- } \\
\text { tes? }\end{array}$} \\
\hline \multicolumn{6}{|c|}{ 2. Secuenciación } \\
\hline \multicolumn{6}{|c|}{ ¿En qué orden trabajarán los contenidos? } \\
\hline \multicolumn{6}{|c|}{ ¿Cómo se organiza cada bloque de contenidos } \\
\hline \multicolumn{2}{|l|}{$[\ldots]$} & \multicolumn{4}{|c|}{$\begin{array}{l}\text { ¿Qué voy a hacer para que el alumno desarrolle el conocimiento X y sea capaz } \\
\text { de usarlo? } \\
\text { Modelo de secuencia }\end{array}$} \\
\hline \multicolumn{3}{|c|}{ Rutinas } & \multicolumn{2}{|c|}{ Metodología general } & Metodología PHE \\
\hline \multicolumn{3}{|l|}{ 1. Localización } & \multicolumn{3}{|c|}{ Situación comunicativa } \\
\hline \multirow[t]{3}{*}{ 2. Desarrollo } & \multicolumn{2}{|c|}{ 2.1. Observación } & \multicolumn{3}{|c|}{ Casos contextualizados } \\
\hline & \multicolumn{2}{|c|}{ 2.2. Explicitación } & \multicolumn{3}{|l|}{ Conocimiento LO } \\
\hline & \multicolumn{2}{|c|}{ 2.3. Contraste } & & & Conocimiento interlingüístico \\
\hline \multirow[t]{2}{*}{ 3.Páctica } & \multicolumn{2}{|c|}{ 3.1. Uso } & \multicolumn{3}{|c|}{ Aplicación contextualizada y significativa del conocimiento } \\
\hline & \multicolumn{2}{|c|}{ 3.2. Corrección } & \multicolumn{3}{|l|}{ Feedback } \\
\hline
\end{tabular}

3. Tipos de actividades y procedimientos

¿Qué actividades y procedimientos voy a utilizar en cada fase

Fase práctica: Uso estratégico del conocimiento lingüístico de LO y del conocimiento interlingüístico

4. Elaboración de materiales

Tabla 8. Modelo de planificación extendido 


\begin{tabular}{|l|l|}
\hline LISTA DE VERIFICACIÓN de secuencia & Respuesta** \\
\hline ¿Se remite a una situación comunicativa en que ocurren las unidades? & \\
\hline $\begin{array}{l}\text { ¿El aprendiz tiene acceso a casos contextualizados para observar el } \\
\text { funcionamiento de la unidad? }\end{array}$ & \\
\hline $\begin{array}{l}\text { ¿Se explicita el funcionamiento del aspecto en la LO, se presentan las } \\
\text { formas, valores o asociaciones necesarias? }\end{array}$ & \\
\hline$¿$ Se presentan o se hacen visibles las diferencias entre las dos lenguas? & \\
\hline $\begin{array}{l}\text { ¿Hay actividades en que se realizan operaciones con los contenidos en } \\
\text { contextos significativos? }\end{array}$ & \\
\hline $\begin{array}{l}\text { ¿Hay actividades en las que se invite a hacer un uso estratégico del } \\
\text { conocimiento metalingüístico e interlingüístico? }\end{array}$ & \\
\hline$¿$ Se contempla la provisión de feedback? & \\
\hline
\end{tabular}

* Sí/No: si la respuesta es negativa, justifíquese con criterios lingüísticos o pedagógicos.

Tabla 9. Lista de verificación para una secuencia de contenido competencial 